\title{
Téoros
}

Revue de recherche en tourisme

\section{Le tourisme gai, un marché porteur pour Montréal}

\section{Pierre Bellerose et Jean-François Perrier}

Volume 19, numéro 2, été 2000

Le tourisme des gais et des lesbiennes

URI : https://id.erudit.org/iderudit/1071965ar

DOI : https://doi.org/10.7202/1071965ar

Aller au sommaire du numéro

Éditeur(s)

Université du Québec à Montréal

ISSN

0712-8657 (imprimé)

1923-2705 (numérique)

Découvrir la revue

Citer cet article

Bellerose, P. \& Perrier, J.-F. (2000). Le tourisme gai, un marché porteur pour Montréal. Téoros, 19(2), 49-51. https://doi.org/10.7202/1071965ar d'utilisation que vous pouvez consulter en ligne.

https://apropos.erudit.org/fr/usagers/politique-dutilisation/ 


\section{LE TOURISME GAI, UN MARCHÉ PORTEUR POUR MONTRÉAL}

\section{Pierre Bellerose et Jean-François Perrier}

Tourisme Montréal, aussi connu sous le nom d'Office des congrès et du tourisme du Grand Montréal, cherche depuis plusieurs décennies à accroître et à développer de nouvelles clientèles. Pour atteindre cet objectif, dans un contexte mouvant de segmentation de clientèles, en 1994 le département de la recherche de Tourisme Montréal a décidé de faire une vaste analyse des différents créneaux porteurs pour la destination touristique qu'est Montréal. Celui identifié comme étant le plus prometteur à l'époque était le créneau du marché de la clientèle gaie et lesbienne. Des recherches ultérieures, effectuées à l'interne ou par des consultants extérieurs, sont venues raffiner la connaissance de ce marché.

Des estimés conservateurs dénombrent environ 16 millions de gais et de lesbiennes en Amérique du Nord qui dépensent près de 47 milliards de dollars en services touristiques seulement. Ce montant représente $10 \%$ du volume total des dépenses pour l'ensemble de l'industrie touristique nord-américaine. En fait, même si la population gaie et lesbienne représente, selon certaines sources, $6 \%$ du total de la population américaine, ceux-ci voyagent en moyenne beaucoup plus que les hétérosexuels. En fait, ils peuvent consacrer une partie plus importante de leurs revenus aux voyages en raison d'obligations familiales souvent moindres. De plus, la facilité relative à rejoindre ce marché le rend très attractif à toute organisation de promotion touristique. À ce chapitre, les premières recherches de 1994 démontraient déjà qu'il s'agissait d'un créneau structuré possédant ses propres associations en tourisme, une multitude de magazines spécialisés et ciblés, des sites Internet (apparus depuis quelques années, ceux-ci se multiplient rapidement) et même un réseau de distribution touristique parallèle en croissance.

Il faut aussi souligner la propension des clientèles homosexuelles américaines à effectuer des voyages internationaux ; en effet, $85 \%$ des gais et des lesbiennes américains prennent des vacances chaque année comparativement à $64 \%$ pour l'ensemble de la population et $45 \%$ de ce segment de marché font des voyages dit « internationaux » contre $9 \%$ pour le reste de la population.

Les différentes sources que les gais et les lesbiennes utilisent pour s' informer sur une destination et pour faire leur choix sont des aspects importants à considérer. Comme l'indique le tableau 1, ces clientèles puisent leurs renseignements de voyages principalement à deux sources : Internet (à un niveau beaucoup plus élevé que la moyenne américaine) et les magazines locaux consacrés aux clientèles gaies et lesbiennes. Il semble que ces clientèles consultent peu les sources généralistes. Par conséquent, il faut les rejoindre principalement par des médias ciblés (traditionnels ou électroniques) auxquels ils peuvent s'identifier. Après avoir colligé l'information, la démarche de Tourisme Montréal a d'abord été de sensibiliser la communauté gaie et de contribuer, dans la mesure du possible, à améliorer le produit qui leur est offert et, parallèlement, de trouver les meilleurs moyens de rejoindre cette communauté (principalement aux États-Unis) afin d'accroître la notoriété de Montréal sur les marchés nord-américains et d'augmenter le nombre de visiteurs.

En fait, depuis une décennie, le produit touristique qui s'adresse spécifiquement à la communauté gaie s'est développé de façon importante à Montréal. On n'a qu'à penser à la trame événementielle (Divers/ Cité, Black \& Blue, Image \& Nation, etc.) ainsi qu'au Village, un des plus importants quartiers gais au monde, où l'on trouve restaurants, cafés, bars, boîtes de nuit, saunas et services. Une telle qualité d'attraits et d'événements peut intéresser fortement la clientèle gaie et lesbienne. Toutefois, il faut continuer d'apporter certaines améliorations à la trame urbaine du Village et, à cet égard, il faut louer les

\begin{tabular}{|c|c|c|}
\hline \multicolumn{3}{|c|}{$\begin{array}{c}\text { TABLEAU 1: } \\
\text { Sources d'information touristique } \\
\text { pour les clientèles gaies et } \\
\text { lesbiennes américaines }\end{array}$} \\
\hline $1-$ & Internet & $65 \%$ \\
\hline $2-$ & Publications gaies locales & $64 \%$ \\
\hline 3- & Guide Damron & $49 \%$ \\
\hline 4- & Publications " bar " locales & $41 \%$ \\
\hline 5- & Magazine The Advocate & $41 \%$ \\
\hline $6-$ & Magazine Out & $38 \%$ \\
\hline 7- & Magazine Genre & $25 \%$ \\
\hline 8- & Travel and Leisure & $25 \%$ \\
\hline 9- & Bulletin Out and About & $24 \%$ \\
\hline $10-$ & Conde Nast Traveler & $21 \%$ \\
\hline
\end{tabular}


efforts de la Chambre de commerce gaie. Notons également que depuis l'été 2000 le Village de Montréal a son kiosque d'information touristique dédié à ce secteur, ce qui ne peut qu'augmenter la qualité du service offert à cette clientèle.

Les études entreprises par Tourisme Montréal ont permis également d'identifier que les gais et les lesbiennes recherchent des destinations sûres, riches en activités culturelles et artistiques, offrant un service hors pair, une gastronomie renommée et la possibilité de rencontrer des gens de leur communauté. En fait, une clientèle qui semble toute désignée pour Montréal !

C'est dans un tel contexte que Tourisme Montréal a commencé, dès 1995, ses premiers efforts de commercialisation visant le marché gai avec l'objectif de répondre aux besoins de cette clientèle et de faire de Montréal l'une des destinations urbaines les plus visitées. Il était alors essentiel d'obtenir la collaboration de plusieurs partenaires et acteurs de l'industrie touristique ainsi que celle de la communauté gaie montréalaise. En effet, pour réussir, au-delà du produit, il faut une volonté et une détermination de tous les intervenants et, surtout, une collectivité prête à appuyer sans réserve la communauté gaie et lesbienne et à développer un produit et des stratégies de promotion conjointes. Tourisme Montréal a trouvé rapidement au sein de l'industrie touristique montréalaise l'enthousiasme nécessaire, et cela dès le début. De plus, les gouvernements du Québec et du Canada ont montré une ouverture certaine face aux revendications de la communauté gaie, dont la reconnaissance des conjoints de fait de même sexe. Cette ouverture gouvernementale était essentielle à la bonne perception de la destination par la communauté gaie américaine.

Ce contexte a permis à Tourisme Montréal d'aller de l'avant et de créer une expérience de voyage qui pourrait distinguer Montréal de la concurrence. Depuis 1995, des liens se sont créés avec la communauté et diverses actions ont été entreprises : reconnaissance de la Chambre de commerce gaie du Québec comme interlocuteur principal pour admettre le Village parmi les quartiers touristiques ; participation de Tourisme Montréal à des foires de voyages ; adhésion à l'IGLTA (International Gay \& Lesbian Travel Association); sollicitation et obtention du congrès annuel de l'IGLTA

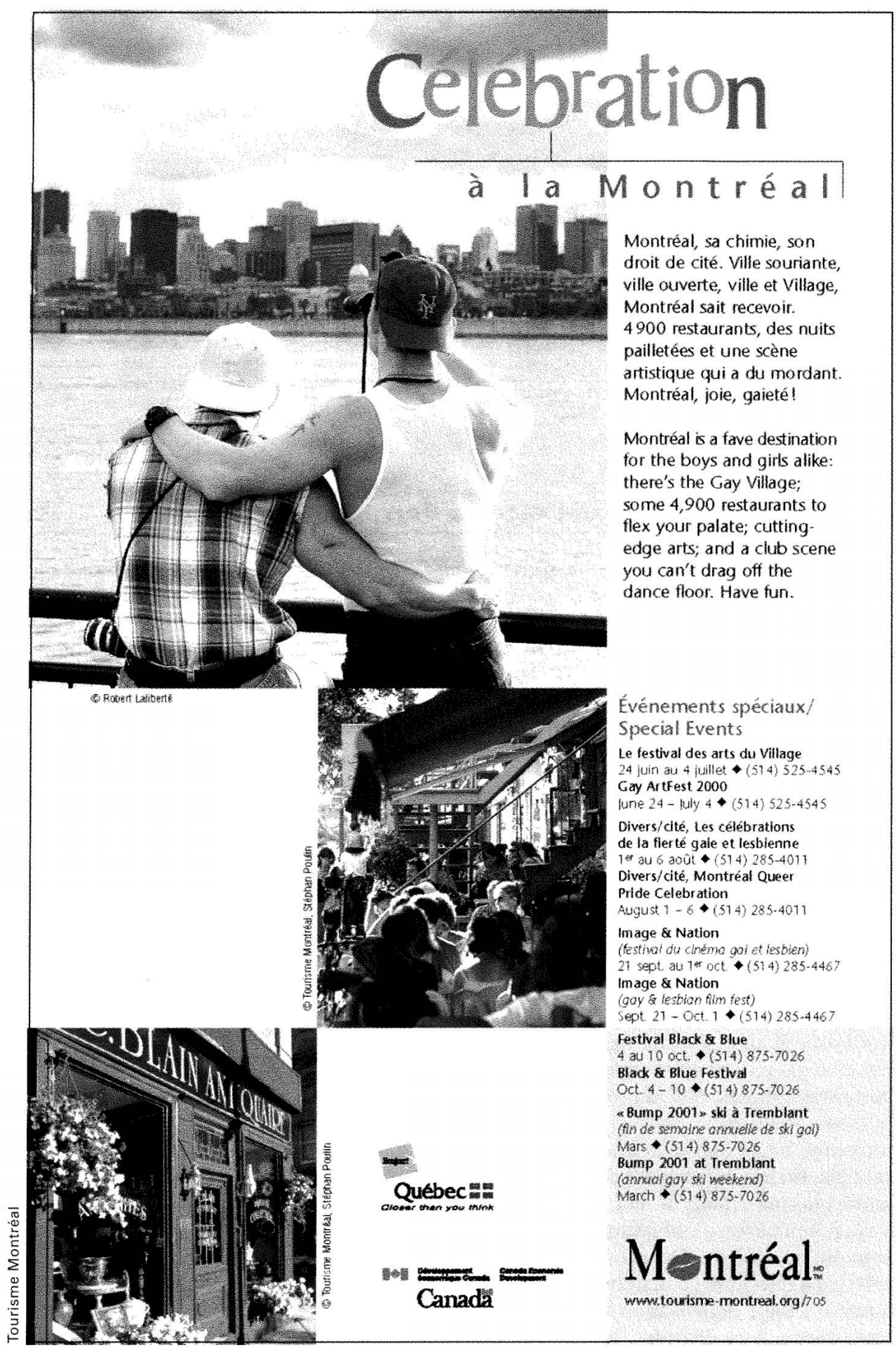

Publicité de Tourisme Montréal destinée au marché gai et lesbien.

en 1998 ; organisation de tournées de presse de journalistes spécialisés ; partenariat avec Divers/Cité et la Fondation Bad Boy Club Montréal ; participation à la candidature de Montréal pour les Jeux Gais 2002 ; accueil de grossistes spécialisés dans ce marché et, en 1997, création de la campagne Visit Gay Friendly Québec avec d'autres partenaires dont Tourisme Québec, à l'attention du marché des États-Unis.

Le mandat de la coordination de la campagne Visit Gay Friendly Québec a été octroyé à une firme de San Francisco, Community Marketing, qui se spécialise dans le tourisme gai. Cette campagne 
d'envergure, en partenariat avec Tourisme Montréal, Tourisme Québec, l'Office des congrès et du tourisme de la communauté urbaine de Québec, Via Rail et Air Canada, a été déterminante dans le positionnement de Montréal et du Québec comme destinations incontournables.

Parmi les actions réalisées, notons: la création d'une image distinctive pour la campagne, publicité ciblée dans plusieurs publications spécialisées, organisation de tournées de presse estivales et hivernales, création d'un site Internet et participation à plusieurs foires de voyages. Le point culminant a été atteint en mars 2000 lors de la Première Conférence internationale sur le tourisme gai tenue à New York. La notoriété de Montréal et du Québec s'accroît rapidement au sein de l'industrie du voyage gai et l'obtention du premier Global Leadership Award par le Gouvernement du Québec, pour sa grande ouverture et sa participation au développement de ce marché, le démontre bien.

Par ailleurs, en novembre 1999, Montréal avait le privilège de se joindre à un cercle restreint de quatre autres villes pour une tournée aux États-Unis à l'intention d'agents de voyage et de grossistes gais. Montréal était alors aux côtés de Paris, de Munich, de Sydney et de Manchester pour faire valoir ses attraits et ses avantages touristiques indéniables.

Un des points culminants des actions de Tourisme Montréal auprès de la clientèle gaie et lesbienne a été sa première participation au défilé de la fierté gaie à San Francisco le 25 juin 2000 . Le thème de la participation de la destination était «Montréal Salutes San Francisco ». Pour l'occasion, on a fait construire et expédier à San Francisco un char allégorique de dimension moyenne, représentant les deux symboles architecturaux des deux villes, soit le Golden Gate Bridge de San Francisco et la Tour du Stade Olympique. De plus, dix-huit danseurs du groupe de mise en forme montréalais Gayrobic ont créé une chorégraphie spéciale. La participation de Montréal à cet événement lui a donné une visibilité importante. En effet, près de 750000 personnes ont assisté au défilé (le plus important au monde selon les organisateurs) qui était également diffusé en direct à la télévision locale. De plus, la couverture en première page du quotidien San Francisco Chronicle de la présence montréalaise assurait à Montréal une reconnaissance comme destination gaie importante. Finalement, Tourisme Montréal y a mérité le prix de la meilleure participation extérieure, soit le Fabulous Visitor Contingent Prize. Ajoutons que Montréal était la seule ville d'envergure à participer au défilé.

À la suite du succès remarquable des dernières années, Tourisme Montréal a confirmé, dans le cadre de sa planification triennale, l'importance des efforts à entreprendre auprès de ce marché. Il est en effet important de préserver et de consolider le leadership que Montréal a acquis face à une concurrence croissante et de mieux en mieux organisée. À cet égard, un gestionnaire de Tourisme Montréal se consacre maintenant presque entièrement à ce marché.

Le plan de développement 2000-2002 peut se résumer ainsi : conserver l'avance acquise depuis 1994, positionner Montréal parmi les cinq premières destinations urbaines au monde, consolider le produit, développer des partenariats avec des grossistes et, bien sûr, augmenter de façon significative le nombre de visiteurs gais et lesbiens.

Déjà, le Village est très bien identifié et décrit dans le guide touristique de Montréal ; le calendrier des événements de la destination mentionne les principales manifestations. De plus, une brochure a été conçue pour décrire la destination et ses attraits et le site Internet de Tourisme Montréal a ajouté des pages qui décrivent le produit gai montréalais. Une nouvelle image est en développement pour les publicités et la banque de photos sera bonifiée. Il est en effet important que les publications touristiques reflètent réellement la communauté gaie et lesbienne montréalaise. Se conjuguera à ces efforts la candidature de Montréal pour l'obtention des Jeux Gais en 2006. Cet événement représenterait une occasion toute spéciale d'accroître substantiellement la notoriété de Montréal sur la scène mondiale. Que ce soit par la publicité, par la présence à de futures Prides ou par la création d'événements spéciaux, une synergie s'installera entre Tourisme Montréal et le Comité de candidature de Montréal 2006 pour optimiser tous les efforts.

D'autres partenaires se joindront à Tourisme Montréal pour diversifier davantage le produit. Par exemple, Tremblant orga- nise depuis la saison 1999-2000 un weekend de ski destiné aux gais, l'Association des commerçants du Village organise le Festival des arts du Village et le Festival Juste pour Rire a inclus dans sa programmation estivale un événement très couru : le Queer Comics.

Il est évident que, depuis 1994, la perception de ce marché a beaucoup évolué au sein de l'industrie touristique montréalaise et Tourisme Montréal a participé à cette évolution. Les études initiales démontraient un créneau en émergence et Tourisme Montréal a fait en sorte que Montréal se démarque rapidement. Les hôtels, les attraits, les événements, les fournisseurs de services, tous se montrent davantage attentifs aux besoins spécifiques des clientèles gaies et lesbiennes. L'ensemble du milieu touristique montréalais doit contribuer à la création de cette nouvelle expérience de voyage. Montréal est désormais la capitale gaie au Canada et Tourisme Montréal souhaite en faire une des destinations gaies incontournables d'Amérique du Nord. L'approche utilisée est très simple, mais rigoureuse : comprendre les clientèles, construire à partir de la réalité (Montréal possédait déjà un quartier gai dynamique et animé) - et non prétendre offrir ce que Montréal n'est pas - et inviter le monde à vivre cette expérience de voyage passionnante et motivante en collaboration avec les intervenants de l'industrie touristique et de la communauté gaie et lesbienne montréalaise.

Pierre Bellerose, diplômé en gestion touristique de l'UQAM, oeuvre à Tourisme Montréal à titre de directeur du développement des affaires et des relations publiques.

Jean-François Perrier est le gestionnaire du développement des marchés gais et lesbiens à Tourisme Montréal. Il détient une formation de guide touristique de l'ITHQ et un baccalauréat en Design de l'environnement de l'UQAM.

\section{BIBLIOGRAPHIE}

Tourisme Montréal (1999), Fiches de marchés potentiels, Montréal, juin.

Tourisme Montréal (1999), Fiche Marketing. Le tourisme gai, Montréal, février, 56 pages et annexes.

Tourisme Montréal (1997), Analyse des marchés potentiels pour Montréal: Fiches synthèses, Montréal, mai, 91 pages. 